\title{
Editorial: Parasitoids' Ecology and Evolution
}

\author{
Catherine Wanjiru Clarke ${ }^{1}$, Paul-André Calatayud ${ }^{2,3 *}$, René F. H. Sforza ${ }^{4}$, \\ Rose Ngeh Ndemah ${ }^{5}$ and Casper Nyamukondiwa ${ }^{6}$
}

${ }^{1}$ Agriculture Victoria Research, Rutherglen, VIC, Australia, ${ }^{2}$ Université Paris-Saclay, CNRS, IRD, UMR Evolution, Génomes, Comportement et Ecologie, Gif-sur-Yvette, France, ${ }^{3}$ International Centre of Insect Physiology and Ecology, Nairobi, Kenya, ${ }^{4}$ European Biological Control Laboratory (USDA-ARS), Montpellier, France, ${ }^{5}$ International Institute of Tropical Agriculture, Yaoundé, Cameroon, ${ }^{6}$ Biological Sciences and Biotechnology, Botswana International University of Science and Technology, Palapye, Botswana

Keywords: biological control, host-associated differentiation, coevolution, genetic structure, environmental change

\section{Editorial on the Research Topic}

\section{Parasitoids' Ecology and Evolution}

Parasitoids are among arthropods that are most widely used in biological control against crop pests, and thus are a significant component of integrated pest management systems. The interaction of parasitoids with the environment (including both hosts insects and plants) have been well-studied for guilds that lay eggs in or on an insect host, followed by larval development, ultimately killing the host (e.g., Godfray, 1994; Kaiser et al., 2017). A better knowledge of parasitoid's biology and ecology is key to successful application of evolutionary principles and determinant to identifying intimate connections with the life history of respective hosts (Roitberg et al., 2001), that often lead to extremely specialized host-parasitoid interactions. Although often overlooked in research and development, ecological and evolutionary considerations are significant to natural control of pests by parasitoids in agricultural systems (e.g., Heimpel and Mills, 2017). The articles in this Research Topic on "parasitoids' ecology and evolution" address fundamental topics in ecology and evolution of parasitoids and their hosts in a context of global changes, i.e., both climate and landscape changes. As in all science disciplines, the purpose of this Research Topic is to showcase current research and unravel new opportunities for future investigations with respect to management of pests by parasitoids.

Why wide-ranging studies on parasitoid's ecology and evolution? Over the years, there has been valid concerns for non-target impacts and the environment in the utilization of biocontrol agents as part of an integrated management strategy. Consequently, the agents are now only released into agricultural systems for regulation of pests following a thorough and extensive host-testing aimed at delimiting the range of candidate biocontrol agents. This approach has undoubtedly made biological control safer and more predictive ecologically. Research focused on host range exclusively, however, lacks a measure of genetic variation in host use and the responses of the respective hosts under different climate scenarios. Subsequently, there remains uncertainties on the co-evolutionary interactions between hosts, parasitoids, plants, and on future establishments.

Parasitoids complete development in other arthropods, mostly insects, leading to their death or sterility and offer an excellent mechanism for natural and sustainable pest control. Though parasitoids may appear generalists, careful ecological studies tell of a hidden complexity with an assemblage of populations having more restricted host ranges. We therefore highlight that 
studying parasitoid diversity may benefit a successful implementation for their use in biological control and ignoring their diversity can be damaging. Both Frayssinet et al., and Bredlau et al. sought to understand the intra- and interspecific plasticity with an aim of enhancing our knowledge on how biocontrol agents can be used for the advancement of integrated pest management in fluctuating host densities and seasonal changes. Tougeron et al. further provided perspectives on the implications for biological control based on the composition of host-parasitoid communities in the climate change context.

Factors associated with poor parasitoid performance are attributable to management practices, e.g., chemical applications, and disturbances in agricultural landscapes (Gurr et al., 2017). Chidawanyika et al. exemplified the effect of climate change as a modifying factor of parasitoid physiology and thereby the effectiveness of biocontrol in diverse agroecosystems. Factors such as habitat fragmentation have been referred to as key disruptors of parasitoid assemblages (Cooke and Roland, 2000) and attributable to loss of connectivity in community assembly. In order to maintain the parasitoid genetic heterogeneity for a robust resilient agricultural systems, Branca et al. highlighted the attention and the need to consider the evolutionary capacity at local and area-wide landscapes. Research should also consider identifying parasitoids that are adaptable to changing climates and agricultural landscapes, and those which are able to cope with host evolution despite many additional biotic and abiotic ecological forces, including reproduction manipulators that would be expected to reduce local adaptation to hosts (Branca et al.; Chidawanyika et al.).

The success of a biocontrol program depends on the foraging efficiency of parasitoids which includes their ability to accurately locate, manipulate, and accept their hosts (Vinson, 1976). For classical and augmentative biological control, locating the target pest depends on the interplay between the parasitoid, the target pest and the crop. Foraging can be enhanced by adult pre-release exposure to target pest and host plant volatiles. de Bruijn et al. showed that persistent memories, such as those formed after several experiences spaced in time, can lead to maladaptive foraging behavior if the contained information becomes unreliable. Kruidhof et al. also demonstrated that studies focusing on plant volatiles may be constrained by a weak response to foraging cues originating from a crop environment, and/or an innate tendency for dispersal upon release. These latter factors lead to declining searching efficiency and subsequently, reduction of parasitism rates, can be overcome by optimizing the parasitoids foraging behavior through parasitoid olfactory conditioning (POC). Parasitoids can be trained to become more efficient in the different phases involved in the process of host searching and host acceptance and POC can thus, result in a "foraging efficacy gain" (Kruidhof et al.).

The success of parasitoid biocontrol agents in addition, depends strongly also on host density and timing of seasonal activities (phenology) (Berryman, 1999; Jeffs and Lewis, 2013). For example, any decline of pest density linked to changes in their host plant characteristic and seasonal availability (e.g., enhanced evasive behavior of the plants) can drastically affect the parasitism rates and then the success of the biological control program (Tomasetto et al.). To overcome this risk of parasitism decline, modifications in parasitoid community composition linked to shifts in diapause expression (reduction or arrest of the use of winter diapause) and to host availability throughout the year can occur (Tougeron et al.). Parasitoids tend to show a preference for ovipositing in the host species in which they developed regardless of host identity and the availability of alternative hosts because switching to novel hosts is initially time consuming and costly (Jones et al., 2015). The costs and the time frame over which these are incurred, may influence host selection behavior and host-parasitoid dynamics in multispecies communities. Frayssinet et al. described here the ability of a parasitoid to switch hosts in fluctuating densities of the preferred host, a strategy that would allow the parasitoid to avoid seasonal population collapses.

The ability of parasitoids to counter host immune defenses and forage for hosts is dependent on host maternal factors and is shaped by co-evolution (Kraaijeveld and Godfray, 1999). Parasitoids learn to associate environmental cues and food, with hosts while foraging and use specific signals to discriminate between hosts and non-host species using chemical compounds to locate and accept their hosts. Bichang'a et al. (2018) showed for the first time that an enzyme from oral secretions of the host plays a key role in host acceptance and oviposition by parasitoids. The molecular variations in this enzyme could explain and account for host-range differences between parasitoid species and the evolutionary processes involved in chemically-mediated host specialization (Bichang'a et al.). Bracovirus genes though different across orders of parasitoids are responsible for immune suppressive abilities. Parasitoids could be responsible for maintaining a reproductive isolation across a broad range of host-food plant sources by the mode of maternal factors expressed and presence of multiple hosts (Bredlau et al.). These authors highlighted here an unexplored study area in biocontrol programs that comprises parasitoid population structure among different host-associated populations, their maternal factors and host plant sources at the landscape level.

\section{CONCLUSION AND FUTURE CHALLENGES}

Articles published in this Research Topic "parasitoids' ecology and evolution" explore the evolutionary aspects of biological control and opens new areas for future research. For classical biological control that involves importation and introduction of agents from their native range, the choice of biocontrol agents could be based on established relationships with the host in the native range or interactive models that predict the effectiveness of the agents in "new associations" accounting for multiple hosts and scenarios of hosts unavailability. With this Research Topic, we aimed to provide a platform for scientists to share their understanding of mechanisms that drive the ecological and evolutionary interactions between parasitoids and their hosts. The excellent contributions are a demonstration of a still active research community in this and provided an up-to-date 
understanding of the intrinsic capacity of parasitoids to adapt in rapidly changing agricultural landscapes.

\section{AUTHOR CONTRIBUTIONS}

P-AC came up with the topic idea. CC, RS, RN, and CN contributed to its description and to the overall organization of the special Research Topic. All editors contributed to the

\section{REFERENCES}

Berryman, A. A. (1999). "The theoretical foundations of biological control," in Theoretical Approaches to Biological Control, eds B. A. Hawkins and H. V. Cornell (Cambridge: Cambridge University Press), 3-21.

Bichang'a, G. B., Da Lage, J. L., Capdevielle-Dulac, C., Zivy, M., Balliau, T., Sambai, K., et al. (2018). $\alpha$-Amylase mediates host accptenace in the braconid parasitoid Cotesia flavipes. J. Chem. Ecol. 44, 1030-1039. doi: 10.1007/s10886-018-1002-9

Cooke, B. J., and Roland, J. (2000). Spatial analysis of large-scale patterns of forest tent caterpillar outbreaks. Ecoscience 7, 410-422. doi: 10.1080/11956860.2000.11682611

Godfray, H. C. J. (1994). Parasitoids, Behavioral and Evolutionary Ecology. Princeton, NJ: Princeton University Press.

Gurr, G. M., Wratten, S. D., Landis, D. A., and You, M. (2017). Habitat management to suppress pest populations: progress and prospects. Ann. Rev. Entomol. 62, 91-109. doi: 10.1146/annurev-ento-031616-035050

Heimpel, G.E., and Mills, N.J. (2017). Biological Control: Ecology and Application. Cambrdge: Cambridge University Press, 386.

Jeffs, C. T., and Lewis, O. T. (2013). Effects of climate warming on host-parasitoid interactions. Ecol. Entomol. 38, 209-218. doi: 10.1111/een.12026

Jones, T. S., Bilton, A. R., Mak, L., and Sait, S. M. (2015). Host switching in a generalist parasitoid: contrasting transient and transgenerational costs associated with novel and original host species. Ecol. Evol. 5, 459-465. doi: $10.1002 /$ ece 3.1333 overseeing of reviews for this special issue. CC wrote the first version of the cover editorial and all editors contributed to its final version.

\section{ACKNOWLEDGMENTS}

We wish to thank all authors of this Research Topic for their contributions.

Kaiser, L., Ode, P., van Nouhuys, S., Calatayud, P.-A., Colazza, S., Cortesero A.-M., et al. (2017). The plant as a habitat for entomophagous insects. Adv. Bot. Res. 81, 179-223. doi: 10.1016/bs.abr.2016. 09.006

Kraaijeveld, A. R., and Godfray, H. C. J. (1999). Geographic patterns in the evolution of resistance and virulence in Drosophila and its parasitoids. Am. Nat. $153,61-74$.

Roitberg, B. D., Boivin, G., and Vet L. E. M. (2001). Fitness, parasitoids, and biological control: an opinion. Can. Entomol. 133, 429-438. doi: 10.4039/Ent133429-3

Vinson, S. B. (1976). Host selection by insect parasitoids. Annu. Rev. Entomol. 21, 109-133.

Conflict of Interest: The authors declare that the research was conducted in the absence of any commercial or financial relationships that could be construed as a potential conflict of interest.

Copyright (c) 2019 Clarke, Calatayud, Sforza, Ndemah and Nyamukondiwa. This is an open-access article distributed under the terms of the Creative Commons Attribution License (CC BY). The use, distribution or reproduction in other forums is permitted, provided the original author(s) and the copyright owner(s) are credited and that the original publication in this journal is cited, in accordance with accepted academic practice. No use, distribution or reproduction is permitted which does not comply with these terms. 